\title{
THE LÉVY-LINDEBERG CENTRAL LIMIT THEOREM IN ORLICZ SPACES $L_{\phi}$
}

\author{
ANNA T. LAWNICZAK
}

\begin{abstract}
An $L_{\phi}(T, \mathscr{F}, m)$-valued random element $X$, where $\Phi\left(t^{1 / 2}\right)$ is equivalent to a concave function, satisfies the Levy-Lindeberg central limit theorem if and only if it is centered and pre-Gaussian; that is, if and only if $E X(t)=0 \mathrm{~m}$-a.e. and $\left\{E X^{2}(t)\right\}^{1 / 2} \in L_{\phi}$.
\end{abstract}

1. Introduction and preliminaries. It is well known that an $L_{p}$-valued random element $X$, for $1 \leqslant p \leqslant 2$, satisfies the Lévy-Lindeberg central limit theorem (CLT) if and only if $X$ is pre-Gaussian (i.e. $E X(t)=0 m$-a.e. and $\left\{E X^{2}(t)\right\}^{1 / 2} \in L_{p}$ ). This fact is a consequence of $L_{p}$ being of cotype 2-see e.g. [1, \$3.8]. Recently Gine [9] proved the above theorem for $L_{p}$-valued random elements $(0<p<1)$. Since $L_{p}$ is a special type of Orlicz space (where $\Phi(t)=t^{p}$ ) it is therefore natural to ask for which class of Orlicz spaces the above theorem is true. In [10] Gorgadze and Tarieladze proved that when $\Phi\left(t^{1 / 2}\right)$ is equivalent to a convex function $\tilde{\Phi}$ (i.e. $\Phi\left(c_{1} t^{1 / 2}\right) \leqslant \tilde{\Phi}(t)$ $\leqslant \Phi\left(c_{2} t^{1 / 2}\right)$ for some $c_{1}, c_{2} \in(0, \infty)$ and all $\left.t>0\right)$, then $L_{\phi}$ is of type 2 , and, therefore, the condition $\left\{E X^{2}(t)\right\}^{1 / 2} \in L_{\Phi}$ is not sufficient to guarantee that the $L_{\phi}$-valued random element $X$ satisfies the CLT. In [10] it was also proved that for a convex function $\Phi$, such that $\Phi\left(t^{1 / 2}\right)$ is equivalent to a concave function $\tilde{\Phi}, L_{\phi}$ is of cotype 2. Therefore [8 and 9], we can expect, and it was conjectured by Giné, that any $L_{\phi}$-valued random element, where $\Phi\left(t^{1 / 2}\right)$ is equivalent to a concave function $\tilde{\Phi}$, satisfies the CLT if and only if it is pre-Gaussian. It is not difficult to realize that under the last assumption about $\Phi$, all $L_{p}$ spaces, $0<p \leqslant 2$, are a special case of the $L_{\phi}$ spaces which are under consideration.

The proof of the Lévy-Lindeberg CLT in $L_{p}, 0<p<1$, in [9] is based on some theorems whose validity is unknown for general $L_{\Phi}$ spaces and also depends on the $p$-homogeneity of the seminorm. The author's proof is based on the correspondence between measures on $L_{\phi}$ spaces and measurable stochastic processes with sample paths in $L_{\phi}$ spaces, and makes no assumption on the $p$-homogeneity of the seminorm.

The author wishes to thank Evarist Gine for many helpful discussions on this topic, and also the referee of the earlier manuscripts for the remark which allowed to a shortening of the proof of the theorem.

Received by the editors February 1, 1983 and, in revised form, March 22, 1983; presented at the AMS Meeting at Denver, 1983.

1980 Mathematics Subject Classification. Primary 60F05, 60G15.

Key words and phrases. Gaussian measure, Gaussian process, Orlicz space, Lèvy-Lindeberg CLT. 
Let $(T, \bar{F}, m)$ be an arbitrary $\sigma$-finite measure space with $\sigma$-algebra $\bar{y}$ and a separable measure $m$. Let $S$ be the space of equivalence classes in $m$ of all real-valued $\mathcal{F}$ measurable functions.

By $\Phi$ let us denote a continuous, nonnegative, nondecreasing function defined for $u \geqslant 0$ such that $\Phi(u)=0$ if and only if $u=0$. We assume additionally that $\Phi(u)$ satisfies the so-called $\Delta_{2}$ condition, i.e., there is a positive constant $k$ such that for any $u, \Phi(2 u) \leqslant k \Phi(u)$. For $x \in S$, let us put

$$
R_{\phi}(x)=\int_{T} \Phi(|x(t)|) m(d t) .
$$

and let $L_{\phi}$ be the set of all $x \in S$ such that $R_{\phi}(a x)<\infty$ for a positive constant $a$. The set $L_{\phi}$ is a linear space under the usual addition and scalar multiplication. Moreover it becomes a complete, separable metric space [14] under the (usually nonhomogeneous) seminorm $\|\cdot\|_{\phi}$ :

$$
\|x\|_{\phi}=\inf \left\{c: c>0, R_{\phi}\left(c^{-1} x\right) \leqslant c\right\} .
$$

The space $\left(L_{\phi},\|\cdot\|_{\phi}\right)$ is called an Orlicz space.

By the same arguments as in [9] we can show that the restrictions concerning the space $(T, \widetilde{F}, m)$ do not influence the generality of the theorem.

For convenience, let us recall the necessary facts concerning probability measures on $\left(L_{\phi}, \mathscr{B}\left(L_{\phi}\right)\right)$, where $\mathscr{B}\left(L_{\phi}\right)$ is the Borel $\sigma$-algebra in $L_{\phi}$.

A. For each probability measure $\mu$ on $\left(L_{\phi}, \mathscr{B}\left(L_{\phi}\right)\right)$, a measurable stochastic process $\zeta=\{\zeta(t): t \in T\}$ can be constructed with simple paths in $L_{\phi}$ such that $\tilde{\zeta}(x)=x \mu$-a.e. $(\tilde{\zeta}$ is the random element generated by the stochastic process $\zeta)$, the induced measure $\mu_{\zeta}$ is equal to $\mu$, and, conversely, each jointly measurable stochastic process $\zeta(t, \omega)$ defined on $T$, with almost all its sample paths in $L_{\phi}$, induces an $L_{\phi}(T, \mathscr{F}, m)$-valued random element [4].

B. Let $\zeta_{i}$ be some measurable stochastic processes with sample paths in $L_{\phi}$, $i=1,2$, and let $\mu_{i}$ denote the measures induced by $\zeta_{i}$ on $\mathscr{S}\left(L_{\phi}\right), i=1,2$. Then $\mu_{1}=\mu_{2}$ if and only if there is a $T_{0} \in \mathscr{F}, m\left(T_{0}\right)=0$, such that the corresponding finite-dimensional distributions of $\zeta_{1}$ and $\zeta_{2}$ based on points $T \backslash T_{0}$ are equal [3].

C. An $L_{\phi}$-valued r.e. $\tilde{\zeta}$ (or p.m. $\mu$ on $\left(L_{\phi}, \mathscr{B}\left(L_{\phi}\right)\right)$ is Gaussian if for any pair of independent copies of $\tilde{\zeta}, X_{1}$ and $X_{2}$, the random elements $X_{1}+X_{2}$ and $X_{1}-X_{2}$ are independent; this definition is equivalent to: the process $\zeta$ with sample paths in $L_{\phi}$ is Gaussian if and only if there exists a measurable subset $T_{0}, m\left(T_{0}\right)=0$, such that for all finite sets $\left\{t_{1}, \ldots, t_{k}\right\} \subset T \backslash T_{0}$ the random vector $\left\langle\zeta\left(t_{1}\right), \ldots, \zeta\left(t_{k}\right)\right\rangle$ is Gaussian [4].

D. Let $\zeta=\{\zeta(t): t \in T\}$ be a measurable Gaussian process and let $\theta(t)=E \zeta(t)$, $K(s, t)=E(\zeta(s)-\theta(s)) \cdot(\zeta(t)-\theta(t))$. Then for almost every $\omega, \zeta(\cdot, \omega) \in L_{\phi}$ if and only if $\theta \in L_{\phi}$ and (for the diagonal of $K$ ) $K^{1 / 2} \in L_{\phi}$. If almost all sample paths of the process $\zeta$ belong to the space $L_{\phi}$ then the measure $\mu_{\zeta}$ induced by $\zeta$ on $\left(L_{\phi}, \mathscr{G}\left(L_{\phi}\right)\right)$ is Gaussian [5].

E. An $L_{\phi}$-valued r.e. $X$ is pre-Gaussian if and only if $\left\{E X^{2}(t)\right\}^{1 / 2} \in L_{\phi}[12]$.

F. Let $Z_{n}, n \in N$, be $L_{\phi}$-valued symmetric Gaussian r.e.'s such that $\mathscr{E}\left(Z_{n}\right) \underset{\mathrm{w}}{\rightarrow} \mathcal{E}(Z)$. Then $Z$ is also Gaussian and symmetric [9]. 
G. Let $M$ be a complete separable metric space and let $Y_{n, \varepsilon}, Z_{n}$, be $M$-valued r.e.'s $(\varepsilon>0, n \in N)$ such that: (a) $\mathcal{L}\left(Y_{n, \varepsilon}\right) \underset{\mathrm{w}}{\rightarrow} \mathcal{L}\left(Y_{\varepsilon}\right)$ for some r.e.'s $Y_{\varepsilon}$; and (b) for each $\varepsilon>0$ there exists $n(\varepsilon)<\infty$ such that for all $n>n(\varepsilon), d_{\mathrm{pr}}\left(Z_{n}, Y_{n, \varepsilon}\right)<\varepsilon$, where $d_{\mathrm{pr}}$ is any distance metrizing convergence in probability. Then $\mathrm{w}-\lim _{n} \mathcal{L}\left(Z_{n}\right)$ and $\mathrm{w}-\lim _{\varepsilon \downarrow 0} \mathscr{L}\left(Y_{\varepsilon}\right)$ exist and are equal (w-lim denotes limit in the weak topology) (Billingsley [2] and Pisier [13]).

H. Let $x_{k}, x \in L_{\phi}$. Then $\left\|x_{k}-x\right\|_{\phi}$ as $k \rightarrow \infty$ if and only if $R\left(\left|x_{k}-x\right|\right) \rightarrow 0$ as $k \rightarrow \infty[14]$.

Proposition. Let $\left\{X_{n}\right\}$ be a sequence of $L_{\phi}$-valued r.e.'s such that:

(1) $\mathfrak{L}\left(X_{n}\right)$ is a tight family of p.m.

(2) There exists a measurable subset $T_{0}, m\left(T_{0}\right)=0$, such that for any finite set $\left\{t_{1}, \ldots, t_{k}\right\} \subset T \backslash T_{0}$,

$$
\mathcal{L}\left(X_{n}\left(t_{1}\right), \ldots, X_{n}\left(t_{k}\right)\right) \underset{\mathrm{w}}{\rightarrow} \mu_{t_{1}, \ldots, t_{k}} .
$$

Then the sequence $\left\{\mathcal{L}\left(X_{n}\right)\right\}$ is weakly convergent to some p.m. $\mu$ on $\left(L_{\phi}, \mathscr{B}\left(L_{\phi}\right)\right)$ and if $\zeta=\{\zeta(t): t \in T\}$ is any measurable stochastic process with almost all sample paths in $L_{\phi}$ such that $\mu_{\zeta}=\mu$, then there exists a measurable subset $T_{1}, m\left(T_{1}\right)=0$, such that for any finite subset $\left\{t_{1}, \ldots, t_{k}\right\} \subset T \backslash T_{1}, \mathcal{L}\left(\zeta\left(t_{1}\right), \ldots, \zeta\left(t_{k}\right)\right)=\mu_{t_{1} \cdots t_{k}}$.

Proof. For the proof it is sufficient to show that there exists a p.m. $\mu$ on $\left(L_{\phi}, \mathscr{B}\left(L_{\phi}\right)\right)$ such that each subsequence of $\left\{\mathscr{L}\left(X_{n}\right)\right\}$ contains a subsequence which is weakly convergent to $\mu$.

Let $\left\{\mathscr{L}\left(X_{m}\right)\right\}$ be an arbitrary subsequence of $\left\{\mathscr{L}\left(X_{n}\right)\right\}$. Since $\left\{\mathscr{L}\left(X_{n}\right)\right\}$ is a tight family, $\left\{\mathcal{L}\left(X_{m}\right)\right\}$ contains a subsequence $\left\{\mathcal{L}\left(X_{m_{l}}\right)\right\}$, such that $\mathcal{L}\left(X_{m_{l}}\right) \underset{\mathrm{w}}{\rightarrow}$, for some p.m. $\gamma$. By Skorohod's theorem there exist $L_{\phi}$-valued random elements $G_{m}, G$ with laws $\mathcal{L}\left(X_{m_{l}}\right), \gamma$, respectively, such that $G_{m_{l}} \rightarrow G$ a.e. This implies that for some subsequence $\left(m_{i}\right), G_{m_{i}}(t, \omega) \rightarrow G(t, \omega)(m \times P)$-a.e. From this and fact B it follows that there exists a measurable subset $T_{0}, m\left(T_{0}\right)=0$, such that for any finite set $\left\{t_{1}, \ldots, t_{k}\right\} \subset T \backslash T_{0}$,

$$
\left\langle G_{m_{i}}\left(t_{1}\right), \ldots, G_{m_{i}}\left(t_{k}\right)\right\rangle \rightarrow\left\langle G\left(t_{1}\right), \ldots, G\left(t_{k}\right)\right\rangle \text { in } P ;
$$

therefore $\mathscr{L}\left(G_{m_{i}}\left(t_{1}\right), \ldots, G_{m_{i}}\left(t_{k}\right)\right) \rightarrow \mathfrak{L}\left(G\left(t_{1}\right), \ldots, G\left(t_{k}\right)\right)$. Since

$$
\mathcal{L}\left(G_{m_{i}}\left(t_{1}\right), \ldots, G_{m_{i}}\left(t_{k}\right)\right)=\mathfrak{L}\left(X_{m_{i}}\left(t_{1}\right), \ldots, X_{m_{i}}\left(t_{k}\right)\right),
$$

then, by assumption (2) of the Proposition,

$$
\mathscr{L}\left(G\left(t_{1}\right), \ldots, G\left(t_{k}\right)\right)=\mu_{t_{1}, \ldots, t_{k}},
$$

and from fact B it follows that there exists a p.m. $\mu$ on $\left(L_{\phi}, \mathscr{B}\left(L_{\phi}\right)\right)$ such that each subsequence of $\left\{\mathcal{L}\left(X_{n}\right)\right\}$ contains a subsequence which converges weakly to $\mu$. Let $\zeta$ be as in the assumption of the Proposition. Then by $\mathbf{B}$ there exists a measurable subset $T_{1}, T_{0} \subset T_{1}, m\left(T_{1}\right)=0$, such that for any finite set $\left\{t_{1}, \ldots, t_{k}\right\} \subset T \backslash T_{1}$,

$$
\mathcal{L}\left(\zeta\left(t_{1}\right), \ldots, \zeta\left(t_{k}\right)\right)=\mathcal{L}\left(G\left(t_{1}\right), \ldots, G\left(t_{k}\right)\right)=\mu_{t_{1}, \ldots, t_{k}} .
$$


2. The Central Limit Theorem. We assume throughout that $\Phi\left(t^{1 / 2}\right)$ is equivalent to a concave function $\tilde{\Phi}$ (i.e. $\Phi\left(c_{1} t^{1 / 2}\right) \leqslant \tilde{\Phi}(t)<\Phi\left(c_{2} t^{1 / 2}\right)$ for some $c_{1}, c_{2} \in(0, \infty)$ and all $t>0)$, and Jensen's inequality implies, for any random variable $\zeta$,

$$
E \tilde{\Phi}(|\zeta|) \leqslant \tilde{\Phi}(E|\zeta|)
$$

THEOREM. (a) Let $X$ be a centered $L_{\phi}=L_{\phi}(T, \mathscr{F}, m)$-valued r.e., $\Phi\left(t^{1 / 2}\right)$ is equivalent to $\tilde{\Phi}$-concave, and let $X_{i}, i \in N$, be independent copies of $X$. If

$$
\left\{E X^{2}(t)\right\}^{1 / 2} \in L_{\phi}
$$

then

$$
\mathcal{L}\left(\sum_{i=1}^{n} \frac{X_{i}}{n^{1 / 2}}\right) \underset{\mathrm{w}}{\rightarrow} \gamma
$$

where $\gamma$ is the symmetric Gaussian p.m. determined by the covariance of $X$, i.e. such that

$$
\int_{L_{\phi}} x(s) x(t) d \gamma(x)=E X(s) X(t)
$$

for all $s$ and $t$ outside a set of m-measure zero.

(b) Conversely, if $X$ is an $L_{\phi}$-valued r.e. such that $\left\{\left[\left(\sum_{i=1}^{n} X_{i} / n^{1 / 2}\right)\right\}_{n=1}^{\infty}\right.$ is weakly convergent, then $X$ is centered and (2) holds (hence also (3) and (4)).

Proof. For the proof of (a), let $T_{r} \nearrow T, r \in N$, with $T_{r} \in \mathscr{F}, m\left(T_{r}\right)<\infty$. Let $\sigma(t)=K^{1 / 2}(t, t)$. For each $T_{r}$ and for each $c>0$ let us define

$$
\begin{gathered}
Y_{c}^{r}(t, \omega)= \begin{cases}X(t, \omega) & \text { if } t \in T_{r} \text { and } \sigma(t)<c, \\
0 & \text { otherwise, }\end{cases} \\
Z_{c}^{r}(t, \omega)=X(t, \omega)-Y_{c}^{r}(t, \omega),
\end{gathered}
$$

and, similarly, $Z_{i, c}^{r}, Y_{i, c}^{r}$ for the r.e.'s $X_{i}$. Clearly $Y_{c}^{r}, Z_{c}^{r}$ are still mean zero r.e.'s. Since

$$
\left\{E\left|Y_{c}^{r}(t, \omega)\right|^{2}\right\}^{1 / 2} \begin{cases}\leqslant c & \text { if } t \in T_{r}, \\ =0 & \text { otherwise, }\end{cases}
$$

this ensures that $Y_{c}^{r}$ satisfies CLT in $L_{2}(T, \mathscr{F}, m)$ and therefore (a fortiori) in $L_{\phi}(T, \mathcal{F}, m)$. Since

$$
\begin{aligned}
& E R_{\phi}\left(c_{1} c_{2}^{-1} n^{-1 / 2} \sum_{i=1}^{n} Z_{i, c}^{r}\right) \leqslant E R_{\tilde{\phi}}\left(c_{2}^{-2} n^{-1}\left(\sum_{i=1}^{n} Z_{i, c}^{r}\right)^{2}\right) \\
& \quad \leqslant R_{\tilde{\phi}}\left(c_{2}^{-2} n^{-1} E\left(\sum_{i=1}^{n} Z_{i, c}^{r}\right)^{2}\right)=R_{\tilde{\phi}}\left(c_{2}^{-2} E\left(Z_{c}^{r}(t)\right)^{2}\right) \\
& \quad \leqslant R_{\phi}\left(\left\{E\left(Z_{c}^{r}(t)\right)^{2}\right\}^{1 / 2}\right)=\int_{T / T_{r}} \Phi(\sigma(t)) m(d t)+\int_{T_{r}} \Phi\left(\sigma(t) 1_{\{t: \sigma(t)>c\}}\right) m(d t) \\
& \quad \rightarrow 0
\end{aligned}
$$

uniformly in $n$ as $r \wedge c=\min (r, c) \rightarrow \infty$, then there exists a subsequence $r^{\prime} \wedge c^{\prime}$, such that

$$
R_{\phi}\left(n^{-1 / 2} \sum_{i=1}^{n} Z_{i, c^{\prime}}^{r^{\prime}}\right) \rightarrow 0
$$


almost everywhere uniformly in $n$ as $r^{\prime} \wedge c^{\prime} \rightarrow \infty$. By fact $\mathrm{H}$,

$$
\left\|n^{-1 / 2} \sum_{i=1}^{n} Z_{i, c^{\prime}}^{r^{\prime}}\right\|_{\Phi} \rightarrow 0
$$

almost everywhere uniformly in $n$ as $r^{\prime} \wedge c^{\prime} \rightarrow \infty$. Since $Y_{c}^{r}$ satisfies CLT in $L_{\phi}(T, \mathcal{F}, m)$, then facts $\mathrm{F}, \mathrm{G}$ and (5) imply

$$
\varrho\left(n^{-1 / 2} \sum_{i=1}^{n} X_{i}\right) \underset{\mathrm{w}}{\rightarrow} \gamma
$$

where $\gamma$ is a symmetric Gaussian p.m. on $\left(L_{\phi}, \mathscr{B}\left(L_{\phi}\right)\right)$. There exists a measurable subset $T_{0}, m\left(T_{0}\right)=0$, such that $E X^{2}(t)<\infty$ for $t \subset T \backslash T_{0}$, and the finite-dimensional CLT implies that for any finite subset $\left\{t_{1}, \ldots, t_{k}\right\} \subset T \backslash T_{0}$,

$$
\mathcal{L}\left(\sum_{i=1}^{n} \frac{X_{i}\left(t_{1}\right)}{n^{1 / 2}}, \ldots, \sum_{i=1}^{n} \frac{X_{i}\left(t_{k}\right)}{n^{1 / 2}}\right) \rightarrow \gamma_{t_{1}, \ldots, t_{k}},
$$

$k$-dimensional Gaussian measure with the covariance function $\left\{E X\left(t_{i}\right) X\left(t_{j}\right): i, j=\right.$ $1, \ldots, k\}$. Then by the Proposition it follows that

$$
\int_{L_{\phi}} x(s) x(t) d \gamma(x)=E X(s) X(t)
$$

for all $s$ and $t$ outside a set of $m$-measure zero.

Proof of (b). Let $X^{\prime}$ be an independent copy of $X$, and let $\tilde{X}=X-X^{\prime}$. Then

$$
\gamma_{n}=\mathcal{L}\left(\sum_{i=1}^{n} \frac{\tilde{X}_{1}}{n^{1 / 2}}\right) \underset{\mathrm{w}}{\gamma}
$$

There exist Gaussian r.e.'s $G_{n}, G$ with laws $\gamma_{n}, \gamma, n \in N$, such that $G_{n} \rightarrow G$ a.e. This implies there exists a subsequence $\left(n^{\prime}\right)$ such that, for almost every $t, G_{n^{\prime}}(t, \omega) \rightarrow$ $G(t, \omega)$ in $P$, therefore in law. By fact B there exists a measurable subset $T_{0}$, $m\left(T_{0}\right)=0$, such that for any finite subset $\left\{t_{1}, \ldots, t_{k}\right\} \subset T \backslash T_{0}$,

$$
\mathcal{L}\left(G_{n^{\prime}}\left(t_{1}\right), \ldots, G_{n^{\prime}}\left(t_{k}\right)\right)=\varrho\left(\sum_{i=1}^{n^{\prime}} \frac{\tilde{X}_{i}\left(t_{i}\right)}{\left(n^{\prime}\right)^{1 / 2}}, \ldots, \sum_{i=1}^{n^{\prime}} \frac{\tilde{X}_{i}\left(t_{k}\right)}{\left(n^{\prime}\right)^{1 / 2}}\right)
$$

and

$$
\mathcal{L}\left(G_{n^{\prime}}\left(t_{1}\right), \ldots, G_{n^{\prime}}\left(t_{k}\right)\right) \underset{\mathrm{w}}{\rightarrow} \mathcal{L}\left(G\left(t_{1}\right), \ldots, G\left(t_{k}\right)\right) .
$$

By the finite-dimensional CLT it follows that for any $\left\{t_{1}, \ldots, t_{k}\right\} \subset T \backslash T_{0}$, $\left(G\left(t_{1}\right), \ldots, G\left(t_{k}\right)\right)$ is a symmetric Gaussian vector with the covariance function $\left\{E \tilde{X}_{i}(t) \tilde{X}\left(t_{j}\right): i, j=1, \ldots, k\right\}$. Then facts $\mathrm{C}$ and $\mathrm{D}$ imply that $\gamma$ is a centered Gaussian measure on $\left(L_{\phi}, \mathfrak{B}\left(L_{\phi}\right)\right)$ such that

$$
\int_{L_{\phi}} x(s) x(t) \gamma(d x)=E \tilde{X}(s) \tilde{X}(t)
$$


for all $s$ and $t$ outside a set of $m$-measure zero and $\left\{E \tilde{X}^{2}(t)\right\}^{1 / 2} \in L_{\boldsymbol{\Phi}}$. Therefore for some $a>0$,

$$
\begin{aligned}
& E R_{\phi}\left(a c_{1} c_{2}^{-1}|X(t)-E X(t)|\right) \leqslant E R_{\tilde{\phi}}\left(a^{2} c_{2}^{-2}|X(t)-E X(t)|^{2}\right) \\
& \quad \leqslant R_{\tilde{\phi}}\left(a^{2} c_{2}^{-2} E(X(t)-E X(t))^{2}\right) \leqslant R_{\phi}\left(a\left\{E(X(t)-E X(t))^{2}\right\}^{1 / 2}\right)<\infty,
\end{aligned}
$$

and the process $X(t)-E X(t)$ has almost all its sample paths in $L_{\Phi}$ so the function (class of functions) $E X(t)$ is in $L_{\phi}$. Since $\left\{E(X(t)-E X(t))^{2}\right\}^{1 / 2} \in L_{\phi}$, by the first part of the theorem $X-E X$ also satisfies the CLT, but this implies the functions $n^{1 / 2} E X(t)$ are $L_{\phi}$ bounded. Hence, $E X(t)=0 m$-a.e. and $\left\{E X^{2}(t)\right\}^{1 / 2} \in L_{\phi}$, hence (b) is proved.

Let us denote by $D_{\phi}=D_{L_{\phi}}[0,1]$ the space of all functions defined on the unit interval into $L_{\phi}$ which are right-continuous and have left-hand limits endowed with the Skorohod topology induced by the metric $d_{0}[2,6]$.

Let $\left\{\xi_{n}\right\}_{n=1}^{\infty}$ be a sequence of i.i.d. $L_{\phi}$-valued random elements, $\Phi\left(t^{1 / 2}\right)$ equivalent to $\tilde{\Phi}$-concave, defined on a common probability space $(\Omega, \Sigma, P)$. Define $S_{0}=0$, $S_{n}=\xi_{1}+\cdots+\xi_{n}$ and $X_{n}(t)=S_{[n t]} / n^{1 / 2}$. Obviously, $X_{n}$ is a sequence of $D_{\phi}$-valued random elements. By applying our theorem to Corollary 1 of [6] we immediately get the necessary and sufficient conditions for the Invariance Principle for $L_{\phi}$-valued random elements $\left(\Phi\left(t^{1 / 2}\right)\right.$ equivalent to $\tilde{\Phi}$-concave).

Corollary 1. The sequence of $D_{\phi}$-valued random elements $X_{n}(t)=S_{[n t]} / n^{1 / 2}$ converges in distribution to the Wiener process $W_{\xi}$ if and only if $\left\{E \xi_{1}^{2}(t)\right\}^{1 / 2} \in L_{\phi}$.

Let $S_{n}$ and $X_{n}$ be defined as before on $(\Omega, \Sigma, P)$. By $\nu_{n}$ let us denote a sequence of integer-valued random variables defined on $(\Omega, \Sigma, P)$, and by $a_{n}$, a sequence of positive reals tending to infinity. Define

$$
Y_{n}=\left(1 / \sqrt{\nu_{n}}\right) S_{[n t]}, \quad Z_{n}=\left(1 / \sqrt{a_{n}}\right) S_{[n t]} .
$$

By Theorems 2 and 3 from [6] and our Theorem we get the following corollary concerning the random change of time.

Corollary 2. Assume $\left\{E \xi^{2}(t)\right\}^{1 / 2} \in L_{\phi}$. If :

(i) $\nu_{n} / a_{n}$ converges in probability to a positive constant $\theta$, then $Y_{n} \underset{\mathrm{w}}{\rightarrow} W_{\xi}$ and $Z_{n \rightarrow \mathrm{w}} \theta^{1 / 2} W_{\xi}$.

(ii) $\nu_{n} / a_{n}$ converges in probability to a positive random variable $\theta$ then $Y_{n} \rightarrow W_{\xi}$ and $Z_{n} \rightarrow \theta_{0}^{1 / 2} W_{\xi}$, where $\theta_{0}$ is independent of $W_{\xi}$ and has the same distribution as $\theta$.

(iii) $\left(1 / \sqrt{\nu_{n}}\right) S_{\nu_{n}} \rightarrow \xi$ and $\left(1 / \sqrt{a_{n}}\right) S_{\nu_{n} \rightarrow \mathrm{w}} \theta_{0}^{1 / 2} \xi$.

\section{REFERENCES}

1. A. Araujo and E. Giné, The central limit theorem for real and Banach valued random variables. Wiley, New York, 1980.

2. P. Billingsley, Convergence of probability measures, Wiley, New York, 1968.

3. T. Byczkowski, Gaussian measures on $L_{p}$ spaces, $0 \leqslant p \leqslant \infty$. Studia Math. 59 (1977), 249-261.

4. __ Some results concerning Gaussian measures on metric linear spaces, Lecture Notes in Math.. vol. 656, Springer-Verlag, 1978, pp. 1-16. 
5. . Norm convergent expansion for $L_{\Phi}$-valued Gaussian random elements, Studia Math. 64 (1979), 87-95.

6. T. Byczkowski and T. Inglot, The invariance principle for vector valued random variables with applications to functional random limit theorems, preprint, 1980.

7. N. Dunford and J. T. Schwartz, Linear operators, Vol. I, Interscience, New York, 1958.

8. E. Gine and J. Zinn, Central limit theorems and weak laws for large numbers in certain Banach spaces, preprint, 1981

9. E. Gine, The Levy-Lindeberg central limit theorem in $L_{p}, 0<p<1$, Proc. Amer. Math. Soc. 88 (1983), 147-153.

10. T. G. Gorgadze and V. I. Tarieladze, On geometry of Orlicz spaces, Lecture Notes in Math., vol. 828 , Springer-Verlag, 1980, pp. 47-52.

11. A. Kufner et al., Function spaces, Noordhoff, Leyden, 1977.

12. A. T. Lawniczak, Gaussian measures on Orlicz spaces and abstract Wiener Spaces, preprint, 1981.

13. G. Pisier, Le théorème limite central et la loi du logarithme itére dans les espaces de Banach, Sém. Maurey-Schwartz (1975-1976), Exp. III et IV.

14. S. Rolewicz, Metric linear spaces, PWN, Warsaw, 1972.

Department of Mathematics, Louisiana State University, Baton Rouge, Louisiana 70803

Current address: Department of Mathematics, University of Michigan, Dearborn, Michigan 48128 